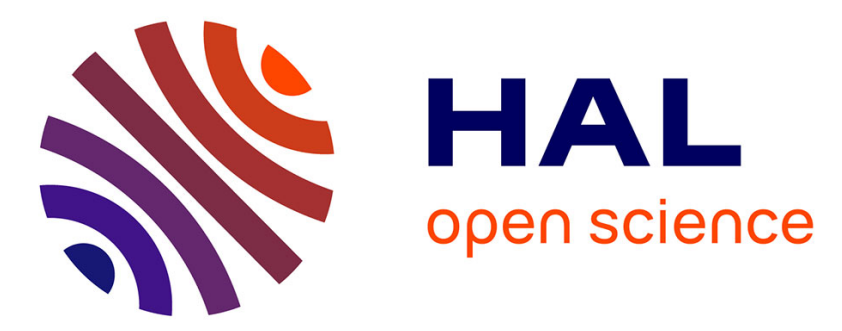

\title{
Explicit formula for a general integral of motion for a class of mechanical systems subject to holonomic constraint
}

J.-W. Perram, Anton Shiriaev, Carlos Canudas de Wit, Frédéric Grognard

\section{- To cite this version:}

J.-W. Perram, Anton Shiriaev, Carlos Canudas de Wit, Frédéric Grognard. Explicit formula for a general integral of motion for a class of mechanical systems subject to holonomic constraint. 2nd IFAC Workshop on Lagrangian and Hamiltonian Methods for Nonlinear Control, Mar 2004, Sevilla, Spain. pp.99-104. hal-01091637

\section{HAL Id: hal-01091637 https://hal.inria.fr/hal-01091637}

Submitted on 5 Dec 2014

HAL is a multi-disciplinary open access archive for the deposit and dissemination of scientific research documents, whether they are published or not. The documents may come from teaching and research institutions in France or abroad, or from public or private research centers.
L'archive ouverte pluridisciplinaire HAL, est destinée au dépôt et à la diffusion de documents scientifiques de niveau recherche, publiés ou non, émanant des établissements d'enseignement et de recherche français ou étrangers, des laboratoires publics ou privés. 


\title{
EXPLICIT FORMULA FOR A GENERAL \\ INTEGRAL OF MOTION FOR A CLASS OF \\ MECHANICAL SYSTEMS SUBJECT TO HOLONOMIC CONSTRAINT ${ }^{1}$
}

\author{
John W. Perram* Anton Shiriaev ${ }^{*, 2}$ \\ Carlos Canudas de Wit ${ }^{* *}$ Frédéric Grognard ${ }^{* * *}$ \\ * The Maersk Institute, University of Southern Denmark, \\ Campusvej 55, DK-5230 Odense $M$ \\ DENMARK \\ ** Laboratoire d'Automatique de Grenoble INPG-ENSIEG, \\ UMR CNRS 5528 BP. 46, 3402, Saint Martin d'Hères \\ FRANCE \\ *** INRIA Sophia-Antipolis- Projet COMORE \\ 2004 route des Lucioles, BP 93 \\ 06902 Sophia-Antipolis Cedex, FRANCE
}

Mechanical systems, conserved quantities, holonomic constraints, zero dynamics

\begin{abstract}
The paper suggests an explicit form of a general integral of motion for the dynamics of a mechanical system with $n$ degrees of freedom, while the system is subject to $n-1$ holonomic constraints. The computation of this integral is given for the cart-pendulum with three different holonomic constraints. Copyright (C) 2003 IFAC
\end{abstract}

\section{INTRODUCTION AND MOTIVATING EXAMPLE}

This paper suggests an explicit formula of a general integral for the dynamical system

$$
\alpha(q) \ddot{q}+\beta(q) \dot{q}^{2}+\gamma(q)=0
$$

where $q \in R^{1} ; \alpha(q), \beta(q)$ and $\gamma(q)$ are smooth scalar functions.

Exploring the properties of system (1) is mainly motivated by the fact that any $n$-degree of freedom mechanical system with $(n-1)$ holonomic

\footnotetext{
1 The work has been supported by the European Commission via the Nonlinear and Adaptive Control network (NACO2) and the Danish Technical Research Council, the grant 26-01-0164

2 The corresponding author
}

constraints, can be rewritten in the form (1). From the point of view of controlling a mechanical system, such holonomic constraints could be seen as 'artificial'; they are forced by an appropriate feedback strategy.

To clarify the last point, consider the ubiquitous cart-pendulum system

$$
M(q) \ddot{q}+C(q, \dot{q}) \dot{q}+G(q)=\tau,
$$

where $q=[x, \theta]^{T} \in R^{1} \times S^{1}, x$ is the horizontal displacement of the cart, $\theta$ is the angle between the pendulum rod and the vertical which is zero at the upright position;

$$
M(q)=\left[\begin{array}{cc}
M+m & m l \cdot \cos \theta \\
m l \cdot \cos \theta & m l^{2}
\end{array}\right],
$$




$$
C(q, \dot{q})=\left[\begin{array}{cc}
0 & -m l \cdot \sin \theta \cdot \dot{\theta} \\
0 & 0
\end{array}\right],
$$

$m, M$ are the masses of the pendulum and the cart respectively; $l$ is the length of the rod;

$$
G(q)=\left[\begin{array}{c}
0 \\
-m g l \cdot \sin \theta
\end{array}\right], \quad \tau=\left[\begin{array}{l}
f \\
0
\end{array}\right]
$$

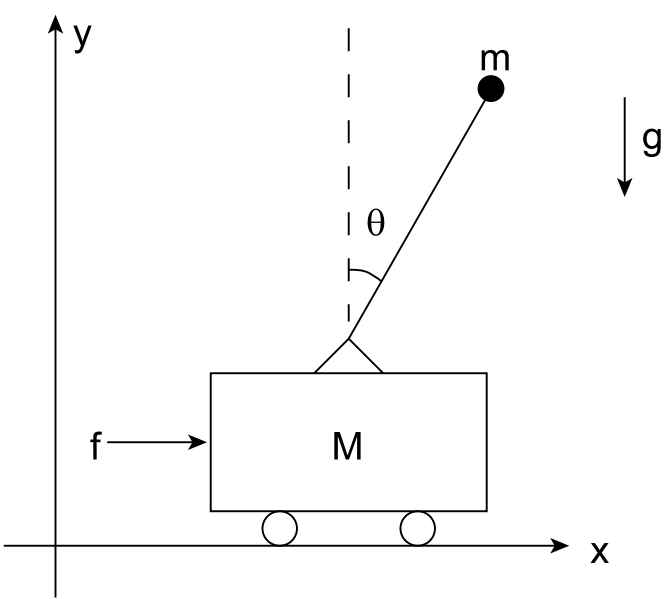

Fig. 1. The cart pendulum system

Suppose that the external control variable $f$ is chosen such that the center of mass of the pendulum is forced to belong to a given vertical line $x_{*}=a$ : the variables $x$ and $\theta$ of the system (2) have to satisfy the constraint

$$
x+l \cdot \sin \theta=a
$$

Taking the first and second time derivatives of (3), one gets two new relations

$$
\begin{array}{r}
\dot{x}+l \cdot \cos \theta \cdot \dot{\theta}=0 \\
\ddot{x}-l \cdot \sin \theta \cdot \dot{\theta}^{2}+l \cdot \cos \theta \cdot \ddot{\theta}=0
\end{array}
$$

As one can expect, the dynamics of (2) subject to the constraint (3) result in a system with 1 degree of freedom. To get its expression, one can substitute the relations (3)-(5) into the second equation of (2), which is

$$
m l \cdot \cos \theta \cdot \ddot{x}+m l^{2} \cdot \ddot{\theta}-m g l \cdot \sin \theta=0
$$

This leads to the following equation with respect to the remaining variable $\theta$

$$
\begin{aligned}
& m l^{2} \cdot \sin ^{2} \theta \cdot \ddot{\theta}+m l^{2} \cdot \cos \theta \cdot \sin \theta \cdot \dot{\theta}^{2}- \\
&-m g l \cdot \sin \theta=0
\end{aligned}
$$

System (7) has the same structure as the general equation (1) with the parameters

$$
\begin{aligned}
& \alpha(\theta)=m l^{2} \cdot \sin ^{2} \theta, \\
& \beta(\theta)=m l^{2} \cdot \cos \theta \cdot \sin \theta, \\
& \gamma(\theta)=-m g l \cdot \sin \theta
\end{aligned}
$$

It will be shown in the next section that, for system (1), there exists a nontrivial function $I$, which preserves its value along any solution of (1). It will be written explicitly, and this is the main contribution of the paper. The orbital stabilization of cycles in the zero-dynamics and in the original system are then discussed as direct consequences of this development. Afterwards two illustrative examples are given.

\section{MAIN RESULT}

Theorem 1. Let $[q(t), \dot{q}(t)]$ be the solution of system (1) with given initial conditions $\left[q_{0}, \dot{q}_{0}\right]$. The function

$$
\begin{gathered}
I\left(q, \dot{q}, q_{0}, \dot{q}_{0}\right)=\dot{q}^{2}-\exp \left\{-2 \int_{q_{0}}^{q} \frac{\beta(\tau)}{\alpha(\tau)} d \tau\right\} \dot{q}_{0}^{2}+ \\
+\exp \left\{-2 \int_{q_{0}}^{q} \frac{\beta(\tau)}{\alpha(\tau)} d \tau\right\} \times \\
\quad \times \int_{q_{0}}^{q} \exp \left\{2 \int_{q_{0}}^{s} \frac{\beta(\tau)}{\alpha(\tau)} d \tau\right\} \frac{2 \gamma(s)}{\alpha(s)} d s
\end{gathered}
$$

is finite and preserves its value along the solution $[q(t), \dot{q}(t)]$

Proof. Observe that for the solution $[q(t), \dot{q}(t)]$ the following identity

$$
\frac{d^{2}}{d t^{2}} q(t)=\frac{d}{d q}\left(\frac{1}{2} \dot{q}^{2}(t)\right)
$$

holds. Indeed,

$$
\begin{aligned}
\frac{d^{2}}{d t^{2}} q(t) & =\frac{d}{d t}(\dot{q}(t)) \\
& =\frac{\partial}{\partial q}(\dot{q}(t)) \cdot \frac{d}{d t}(q(t))=\frac{\partial}{\partial q}(\dot{q}(t)) \cdot \dot{q}(t) \\
& =\frac{d}{d q}\left(\frac{1}{2} \dot{q}^{2}(t)\right)
\end{aligned}
$$

Introducing the new variable

$$
Y=\dot{q}^{2}(t)
$$

and taking (9) into account, one can rewrite the differential equation (1) in the equivalent form

$$
\alpha(q) \frac{1}{2} \frac{d}{d q} Y+\beta(q) Y+\gamma(q)=0
$$


This is a linear equation with respect to function $Y$ and with $q$ (instead of $t$ ) as independent variable.

Let us first consider the case where, along the solution $[q(t), \dot{q}(t)], \alpha(q)$ is separated from zero. Under this assumption, one can divide equation (10) by $\alpha(q)$ and consider the equation

$$
\frac{d}{d q} Y+\frac{2 \beta(q)}{\alpha(q)} Y+\frac{2 \gamma(q)}{\alpha(q)}=0
$$

Its general solution looks as follows

$$
\begin{aligned}
& Y(q)=\exp \left\{-2 \int_{q_{0}}^{q} \frac{\beta(\tau)}{\alpha(\tau)} d \tau\right\} \cdot Y\left(q_{0}\right)- \\
& -\exp \left\{-2 \int_{q_{0}}^{q} \frac{\beta(\tau)}{\alpha(\tau)} d \tau\right\} \times \\
& \quad \times \int_{q_{0}}^{q} \exp \left\{2 \int_{q_{0}}^{s} \frac{\beta(\tau)}{\alpha(\tau)} d \tau\right\} \frac{2 \gamma(s)}{\alpha(s)} d s
\end{aligned}
$$

Introducing function $I$ as

$$
\begin{aligned}
& I\left(q, \dot{q}, q_{0}, \dot{q}_{0}\right)= \\
& Y(q)-\exp \left\{-2 \int_{q_{0}}^{q} \frac{\beta(\tau)}{\alpha(\tau)} d \tau\right\} \cdot Y\left(q_{0}\right)+ \\
& +\exp \left\{-2 \int_{q_{0}}^{q} \frac{\beta(\tau)}{\alpha(\tau)} d \tau\right\} \times \\
& \quad \times \int_{q_{0}}^{q} \exp \left\{2 \int_{q_{0}}^{s} \frac{\beta(\tau)}{\alpha(\tau)} d \tau\right\} \frac{2 \gamma(s)}{\alpha(s)} d s
\end{aligned}
$$

results in $I=0$ along the solution $[q(t), \dot{q}(t)]$. This proves the case when $\alpha(q)$ is separated from zero along the solution $[q(t), \dot{q}(t)]$.

Suppose now that there exists a finite time moment $T_{*}$ such that:

(a) the solution $[q(t), \dot{q}(t)]$ is well defined and remains continuous on the interval $\left[T_{*}-\varepsilon, T_{*}+\varepsilon\right]$ for some $\varepsilon>0$;

(b) the function $\alpha(q)$ equals to zero at $q=q\left(T_{*}\right)$, that is,

$$
\alpha\left(q\left(T_{*}\right)\right)=0 .
$$

Under these assumptions one cannot write the descriptor equation (10) in the form (11) along the solution $[q(t), \dot{q}(t)]$ for the whole time interval
$\left[T_{*}-\varepsilon, T+\varepsilon\right]$. Rather one should consider two equations

$$
\begin{aligned}
& \alpha(q) \ddot{q}+\beta(q) \dot{q}^{2}+\gamma(q)=0, t \in\left[T_{*}-\varepsilon, T_{*}\right) \\
& \alpha(q) \ddot{q}+\beta(q) \dot{q}^{2}+\gamma(q)=0, t \in\left(T_{*}, T_{*}+\varepsilon\right]
\end{aligned}
$$

and one matching condition

$$
\lim _{t \rightarrow T_{*-}}[q(t), \dot{q}(t)]=\lim _{t \rightarrow T_{*+}}[q(t), \dot{q}(t)],
$$

where $t \rightarrow T_{*-}$ is the abbreviation for "approaching $T_{*}$ from the left", and $t \rightarrow T_{*+}$ "from the right". The equality (15) is valid due to assumption (a) of continuity of the solution on the interval $\left[T_{*}-\varepsilon, T+\varepsilon\right]$. This assumption also implies that $[q(t), \dot{q}(t)]$ remains bounded on the interval $\left[T_{*}-\varepsilon, T+\varepsilon\right]$.

For systems (13) and (14), one could write (12) for the subintervals $\left[T_{*}-\varepsilon, T_{*}\right)$ and $\left(T_{*}, T_{*}+\varepsilon\right]$, while the matching condition (15) and boundedness of the solution provide that the function

$$
\begin{aligned}
\dot{q}^{2}(t)= & \exp \left\{-2 \int_{q_{0}}^{q(t)} \frac{\beta(\tau)}{\alpha(\tau)} d \tau\right\} \cdot \dot{q}_{0}^{2}- \\
& -\exp \left\{-2 \int_{q_{0}}^{q(t)} \frac{\beta(\tau)}{\alpha(\tau)} d \tau\right\} \times \\
& \times \int_{q_{0}}^{q(t)} \exp \left\{2 \int_{q_{0}}^{s} \frac{\beta(\tau)}{\alpha(\tau)} d \tau\right\} \frac{2 \gamma(s)}{\alpha(s)} d s
\end{aligned}
$$

remains continuous for all $t \in[0, T+\varepsilon]$. This finishes the proof.

Let us discuss this result:

(1) Function (8) is not the first integral of system (1). Indeed, following the definition, a first integral of a system

$$
\frac{d}{d t} y=f(t, y)
$$

is a function $U=U(t, y)$ that preserves its value along any solution $y(t)$ of the system, see (Hartman, 1964). As shown in the Theorem, I preserves its value along the solutions, but it depends on the initial conditions $\left[q_{0}, \dot{q}_{0}\right]$. On the other hand, such a function is known as a general or full integral of the system. In turn, one can expect that, in many cases, this function leads to the explicit form 
of the first integral of the system. This point is illustrated in the next section, where the cart-pendulum system is presented.

(2) The knowledge of the explicit form of function (8) may be useful when the dynamics of the constraint system exhibits periodic motions for some initial conditions. Choosing 'interesting' initial conditions $\left[q_{0 d}, \dot{q}_{0 d}\right]$, one can use a control Lyapunov function candidate of the form

$$
V(q, \dot{q})=\frac{1}{2} I^{2}\left(q, \dot{q}, q_{0 d}, \dot{q}_{0 d}\right)
$$

for the orbital stabilization of a periodic solution corresponding to the choice $\left[q_{0 d}, \dot{q}_{0 d}\right]$.

\section{ORBITAL STABILIZATION OF CYCLES IN ZERO DYNAMICS}

Suppose that, for some initial conditions $\left[q_{0}^{d}, \dot{q}_{0}^{d}\right]$, the corresponding solution of system (1) is a cycle $\Gamma$. In order to orbitally stabilize this cycle in the controlled system

$$
\alpha(q) \ddot{q}+\beta(q) \dot{q}^{2}+\gamma(q)=u
$$

we can proceed as follows: Choose a Lyapunov function candidate

$$
V(q, \dot{q})=\frac{1}{2} I\left(q, \dot{q}, q_{0}^{d}, \dot{q}_{0}^{d}\right)^{2}
$$

Its time derivative along any solution $[q(t), \dot{q}(t)]$ of system (16) has the form

$$
\begin{aligned}
& \frac{d}{d t} V=I\left(q(t), \dot{q}(t), q_{0}^{d}, \dot{q}_{0}^{d}\right)\left(\frac{\partial I}{\partial q} \dot{q}(t)+\frac{\partial I}{\partial \dot{q}} \ddot{q}(t)\right) \\
& =I \times\left(\frac{\partial I}{\partial q} \dot{q}(t)+\right. \\
& \left.+\frac{\partial I}{\partial \dot{q}}\left[-\frac{\beta(q(t))}{\alpha(q(t))} \dot{q}^{2}(t)-\frac{\gamma(q(t))}{\alpha(q(t))}\right]+\frac{\partial I}{\partial \dot{q}} \frac{u}{\alpha(q(t))}\right) \\
& =-k\left[I\left(q(t), \dot{q}(t), q_{0}^{d}, \dot{q}_{0}^{d}\right) \times \dot{q}(t)\right]^{2} \leq 0
\end{aligned}
$$

where the controller $u=u_{\text {good }}$ is defined to force the last equality:

$$
\begin{aligned}
& u_{\text {good }}=-\frac{k}{2} I\left(q(t), \dot{q}(t), q_{0}^{d}, \dot{q}_{0}^{d}\right) \dot{q}(t) \alpha(q(t))+\quad(19) \\
& +\beta(q(t))\left\{\dot{q}^{2}(t)-\exp \left(-2 \int_{q_{0}^{d}}^{q(t)} \frac{\beta(\tau)}{\alpha(\tau)} d \tau\right) \times\right. \\
& \left.\times\left(\left(\dot{q}_{0}^{d}\right)^{2}-\int_{q_{0}^{d}}^{q(t)} \exp \left\{2 \int_{q_{0}^{d}}^{s} \frac{\beta(\tau)}{\alpha(\tau)} d \tau\right\} \frac{2 \gamma(s)}{\alpha(s)} d s\right)\right\}
\end{aligned}
$$

with some positive parameter $k$.
Under appropriate conditions, the following statement is true: Consider system (1) and one of its cycles $\Gamma$. Choose any point

$$
\left[q_{0}^{d}, \dot{q}_{0}^{d}\right] \in \Gamma
$$

Then $\Gamma$ is an orbitally asymptotically stable cycle of the closed loop system (16), (19).

\section{ORBITAL STABILIZATION OF CYCLES FOR THE ORIGINAL SYSTEM}

In the previous section a procedure for the stabilization of a cycle in the zero dynamics of the system has been proposed. In fact, controller (19) is not defined in the case where the system does not satisfy the holonomic constraint. Suppose that the dynamics of the original mechanical system can be rewritten as follows

$$
\begin{aligned}
& \ddot{q}=-\frac{\beta(q)}{\alpha(q)} \dot{q}^{2}-\frac{\gamma(q)}{\alpha(q)}+\frac{G(q, \dot{q}, y, \dot{y}, v)}{\alpha(q)} \\
& \ddot{y}=-k_{1} y-k_{2} \dot{y}+v
\end{aligned}
$$

where $v$ is a control variable, and

$$
G(q, \dot{q}, y, \dot{y}, v)=0, \quad \text { if } \quad y=\dot{y}=v=0
$$

where $y$ designates the configuration variables not present in the state of the zero-dynamics (possibly after some feedback transformation).

Suppose again that we are given a cycle $\Gamma$ of subsystem (20) (characterized by some $\left.\left[q_{0}^{d}, \dot{q}_{0}^{d}\right]\right)$, and that we are asked to orbitally stabilize this cycle. To proceed we can utilize the back-stepping idea: Consider a Lyapunov function candidate

$$
\begin{aligned}
W(q, \dot{q}, y, \dot{y}) & =\frac{1}{2} I\left(q, \dot{q}, q_{0}^{d}, \dot{q}_{0}^{d}\right)^{2}+ \\
+ & \frac{\varepsilon}{2}\left(G(q, \dot{q}, y, \dot{y}, v)-u_{\text {good }}\right)^{2}
\end{aligned}
$$

where $u_{\text {good }}$ is defined in (19). Taking the time derivative of $W$ along any solution of system (20)(21), we get

$$
\begin{aligned}
& \frac{d}{d t} W=\varepsilon\left(G(q, \dot{q}, y, \dot{y}, v)-u_{\text {good }}\right) \times \\
& \times \frac{d}{d t}\left(G(q, \dot{q}, y, \dot{y}, v)-u_{\text {good }}\right)+I\left(q, \dot{q}, q_{0}^{d}, \dot{q}_{0}^{d}\right) \times \\
& \times\left(\frac{\partial I}{\partial q} \dot{q}(t)-\frac{\partial I}{\partial \dot{q}}\left[\frac{\beta(q)}{\alpha(q)} \dot{q}^{2}+\frac{\gamma(q)}{\alpha(q)}\right]+\frac{\partial I}{\partial \dot{q}} \frac{G}{\alpha(q)}\right) \\
& =\varepsilon\left(G-u_{\text {good }}\right) \times \frac{d}{d t}\left(G-u_{\text {good }}\right)- \\
& \quad-k\left[I\left(q, \dot{q}, q_{0}^{d}, \dot{q}_{0}^{d}\right) \times \dot{q}\right]^{2}+ \\
& \quad+I\left(q, \dot{q}, q_{0}^{d}, \dot{q}_{0}^{d}\right) \times 2 \dot{q} \times \frac{G-u_{\text {good }}}{\alpha(q(t))}
\end{aligned}
$$




$$
\begin{aligned}
= & -k\left[I\left(q, \dot{q}, q_{0}^{d}, \dot{q}_{0}^{d}\right) \times \dot{q}\right]^{2}+\left(G-u_{\text {good }}\right) \times \\
& \times\left(\varepsilon \frac{d}{d t} G-\varepsilon \frac{d}{d t} u_{\text {good }}+I\left(q, \dot{q}, q_{0}^{d}, \dot{q}_{0}^{d}\right) \times \frac{2 \dot{q}}{\alpha(q)}\right)
\end{aligned}
$$

If we choose the control variable $v$ as a solution of the equation

$$
\begin{gathered}
\varepsilon \frac{d}{d t} G(q, \dot{q}, y, \dot{y}, v)-\varepsilon \frac{d}{d t} u_{\text {good }}+ \\
+I\left(q, \dot{q}, q_{0}^{d}, \dot{q}_{0}^{d}\right) \times \frac{2 \dot{q}}{\alpha(q)}= \\
=-\rho\left(G(q, \dot{q}, y, \dot{y}, v)-u_{\text {good }}\right)
\end{gathered}
$$

with some $\rho>0$, then the time derivative of $W$ takes the form

$$
\begin{aligned}
\frac{d}{d t} W=- & k\left[I\left(q, \dot{q}, q_{0}^{d}, \dot{q}_{0}^{d}\right) \times \dot{q}\right]^{2}- \\
- & \rho\left(G(q, \dot{q}, y, \dot{y}, v)-u_{\text {good }}\right)^{2}
\end{aligned}
$$

The last equality (25) implies that the function $W$ is not increasing along the solution of the closed lop system (20), (21), (24), (19). The function $W$ may not be proper even locally around the cycle $\Gamma$. Therefore, convergence of solutions of the closed loop system to the cycle $\Gamma$ remains unproven in general (while it could be proven in some examples).

\section{EXAMPLE: THE CART-PENDULUM}

In this section, we analyze the effect of different holonomic constraints on the zero-dynamics and the general integral of the cart-pendulum.

\subsection{The Center of Mass of the Pendulum Belongs to a Given Vertical}

This example has been discussed in the Introduction, and the constrained dynamics of the system is covered by equation (7). Straightforward calculations, based on formula (8), result in the following expression

$$
\begin{array}{r}
I\left(\theta, \dot{\theta}, \theta_{0}, \dot{\theta}_{0}\right)=\dot{\theta}^{2}-\left[\frac{\sin \theta_{0}}{\sin \theta}\right]^{2} \dot{\theta}_{0}^{2}+ \\
+\frac{2 g}{l \cdot \sin ^{2} \theta}\left[\cos \theta-\cos \theta_{0}\right]
\end{array}
$$

Theorem 1 implies that this function remains zero along the solution $[\theta(t), \dot{\theta}(t)]$ that starts at $\left[\theta_{0}, \dot{\theta}_{0}\right]$. Therefore, function

$$
\begin{aligned}
\sin ^{2} \theta \cdot I & \left(\theta, \dot{\theta}, \theta_{0}, \dot{\theta}_{0}\right)=\sin ^{2} \theta \cdot \dot{\theta}^{2}- \\
& -\sin ^{2} \theta_{0} \cdot \dot{\theta}_{0}^{2}+\frac{2 g}{l}\left[\cos \theta-\cos \theta_{0}\right]
\end{aligned}
$$

will also be zero along the solution. Introducing a function

$$
U(\theta, \dot{\theta})=\sin ^{2} \theta \cdot \dot{\theta}^{2}+\frac{2 g}{l} \cos \theta
$$

one gets

$$
\sin ^{2} \theta \cdot I\left(\theta, \dot{\theta}, \theta_{0}, \dot{\theta}_{0}\right)=U(\theta, \dot{\theta})-U\left(\theta_{0}, \dot{\theta}_{0}\right)
$$

From the last relation one can conclude that $U$ is a first integral of system (7).

\subsection{A Fixed Point on the Pendulum's Rod Belongs to a Given Vertical}

To extend the previous example, consider again the constraint

$$
x+L_{u} \cdot \sin \theta=a
$$

where $L_{u}$ is a fixed parameter (possibly different from the distance $l$ to the center of mass of the pendulum). Taking the first and second timederivatives of (28), one gets

$$
\begin{array}{r}
\dot{x}+L_{u} \cdot \cos \theta \cdot \dot{\theta}=0 \\
\ddot{x}-L_{u} \cdot \sin \theta \cdot \dot{\theta}^{2}+L_{u} \cdot \cos \theta \cdot \ddot{\theta}=0
\end{array}
$$

The substitution of $\ddot{x}$ from the second relation into (6) results in the zero-dynamics

$$
\begin{aligned}
& {\left[l-L_{u} \cdot \cos ^{2} \theta\right] \cdot \ddot{\theta}+} \\
& \quad+L_{u} \cdot \cos \theta \cdot \sin \theta \cdot \dot{\theta}^{2}-g \cdot \sin \theta=0
\end{aligned}
$$

The straightforward calculation of formula (8) results in the following expression

$$
\begin{gathered}
I\left(\theta, \dot{\theta}, \theta_{0}, \dot{\theta}_{0}\right)=\dot{\theta}^{2}-\left|\frac{l-L_{u} \cdot \cos ^{2} \theta_{0}}{l-L_{u} \cdot \cos ^{2} \theta}\right| \dot{\theta}_{0}^{2}- \\
-\frac{2 g}{\left|l-L_{u} \cos ^{2} \theta\right|} \int_{\theta_{0}}^{\theta} \operatorname{sign}\left\{l-L_{u} \cos ^{2} s\right\} \sin s d s
\end{gathered}
$$

If one assumes that along the solution $[\theta(t), \dot{\theta}(t)]$ the function

$$
l-L_{u} \cdot \cos ^{2} \theta(t)>0
$$

then, along this solution, the formula (32) could be rewritten as

$$
\begin{aligned}
I\left(\theta, \dot{\theta}, \theta_{0}, \dot{\theta}_{0}\right)=\dot{\theta}^{2}- & \frac{l-L_{u} \cdot \cos ^{2} \theta_{0}}{l-L_{u} \cdot \cos ^{2} \theta} \dot{\theta}_{0}^{2}+ \\
& +\frac{2 g\left[\cos \theta_{0}-\cos \theta\right]}{l-L_{u} \cdot \cos ^{2} \theta}
\end{aligned}
$$


Theorem 1 indicates that this function remains equal to zero along the solution. Therefore, the function

$$
\begin{aligned}
& \left(l-L_{u} \cdot \cos ^{2} \theta\right) \cdot I\left(\theta, \dot{\theta}, \theta_{0}, \dot{\theta}_{0}\right)= \\
& =\underbrace{\left(l-L_{u} \cdot \cos ^{2} \theta\right) \dot{\theta}^{2}+2 g \cos \theta}_{U(\theta, \dot{\theta})}- \\
& \quad-\left(l-L_{u} \cdot \cos ^{2} \theta_{0}\right) \dot{\theta}_{0}^{2}+2 g \cos \theta_{0} \\
& =U(\theta, \dot{\theta})-U\left(\theta_{0}, \dot{\theta}_{0}\right)
\end{aligned}
$$

will also be zero along the solution, while $U$ is the first integral. It is interesting to observe that, if the parameter $L_{u}$ is chosen as

$$
L_{u}>l
$$

then the upright equilibrium of system (31) is neutrally stable, and the system possesses stable cycles around the upright equilibrium.

\subsection{Constraints without Physical Interpretation}

In the previous examples, the computation of general integrals of the system can easily be transfered to corresponding first integrals. This is not the case in general. To observe this consider the constraint

$$
x+L_{u} \cdot \sin \theta=\varepsilon \cdot \sin (k \theta),
$$

which is a modified version of (28) with new parameters $\varepsilon$ and $k$. Taking the first and second time-derivatives of (28), one gets

$$
\begin{aligned}
& \dot{x}+L_{u} \cdot \cos \theta \cdot \dot{\theta}=\varepsilon \cdot k \cdot \cos (k \theta) \cdot \dot{\theta} \\
& \ddot{x}-L_{u} \cdot \sin \theta \cdot \dot{\theta}^{2}+L_{u} \cdot \cos \theta \cdot \ddot{\theta}= \\
& \varepsilon \cdot k \cdot \cos (k \theta) \cdot \ddot{\theta}-\varepsilon \cdot k^{2} \sin (k \theta) \cdot \dot{\theta}^{2}
\end{aligned}
$$

Substitution of $\ddot{x}$ from the second relation into (6) results in the dynamics

$$
\begin{aligned}
& {\left[l+\varepsilon \cdot k \cdot \cos (k \theta) \cdot \cos \theta-L_{u} \cdot \cos ^{2} \theta\right] \cdot \ddot{\theta}+} \\
& +\left[L_{u} \cdot \cos \theta \cdot \sin \theta-\varepsilon \cdot k^{2} \cdot \sin (k \theta) \cdot \cos \theta\right] \cdot \dot{\theta}^{2}- \\
& \quad-g \cdot \sin \theta=0
\end{aligned}
$$

For this system the function $I$ in (8) looks as follows

$$
\begin{aligned}
& I\left(\theta, \dot{\theta}, \theta_{0}, \dot{\theta}_{0}\right)=\dot{\theta}^{2}- \\
& -\exp \left\{-2 \int_{\theta_{0}}^{\theta} \frac{L_{u} \cos \tau \sin \tau-\varepsilon k^{2} \sin (k \tau) \cos \tau}{l+\varepsilon k \cos (k \tau) \cos \tau-L_{u} \cos ^{2} \tau} d \tau\right\} \dot{\theta}_{0}^{2}+
\end{aligned}
$$

$$
\begin{aligned}
& +\exp \left\{-2 \int_{\theta_{0}}^{\theta} \frac{L_{u} \cos \tau \sin \tau-\varepsilon k^{2} \sin (k \tau) \cos \tau}{l+\varepsilon k \cos (k \tau) \cos \tau-L_{u} \cos ^{2} \tau} d \tau\right\} \times \\
& \times \int_{\theta_{0}}^{\theta} \exp \left\{2 \int_{\theta_{0}}^{s} \frac{L_{u} \cos \tau \sin \tau-\varepsilon k^{2} \sin (k \tau) \cos \tau}{l+\varepsilon k \cos (k \tau) \cos \tau-L_{u} \cos ^{2} \tau} d \tau\right\} \times \\
& \times \frac{-2 g \cdot \sin s}{l+\varepsilon k \cos (k s) \cos s-L_{u} \cos ^{2} s} d s
\end{aligned}
$$

The direct integration inside the last formula may be difficult for different values of parameters $L_{u}, \varepsilon$, $k$, and the form of the corresponding first integral, if it exists, is not straightforward, as it was in the previous examples. Nevertheless, this expression could be used for analysis and control of the system.

\section{CONCLUSIONS}

This paper is aimed at a development of a new method for construction and local orbital stabilization of cycles in mechanical systems. The main idea comes from the fact that we were able to explicitly build a a general integral of motion for any mechanical system with configuration space of dimension $n$ with $(n-1)$ holonomic constraints.

The dynamics of such a system could be reduced to a system with one degree of freedom. If such a system has a cycle, then the explicit form of its general integral of motion enables us to describe this cycle as a zero-level set of the square of this integral function, and use it as a Lyapunov function for deriving a controller and checking orbital asymptotic stability of this cycle in the closed loop system.

For the stabilization of this cycle in the original $n$-degree of freedom mechanical system, an algorithm based on the extension of the back-stepping idea, has been suggested.

\section{REFERENCES}

Hartman Ph. Ordinary Differential Equations, John Wiley \& Sons, 1964. 